The Association

ACAMH for Child and Adolescent Mental Health

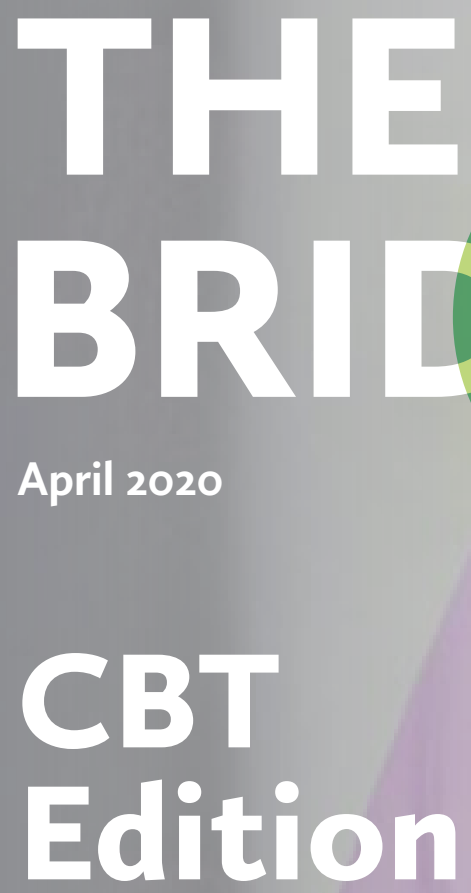

Can transdiagnostic CBT improve outcomes in children with ASD? Does online CBT wo for treating adolescent anxiety?
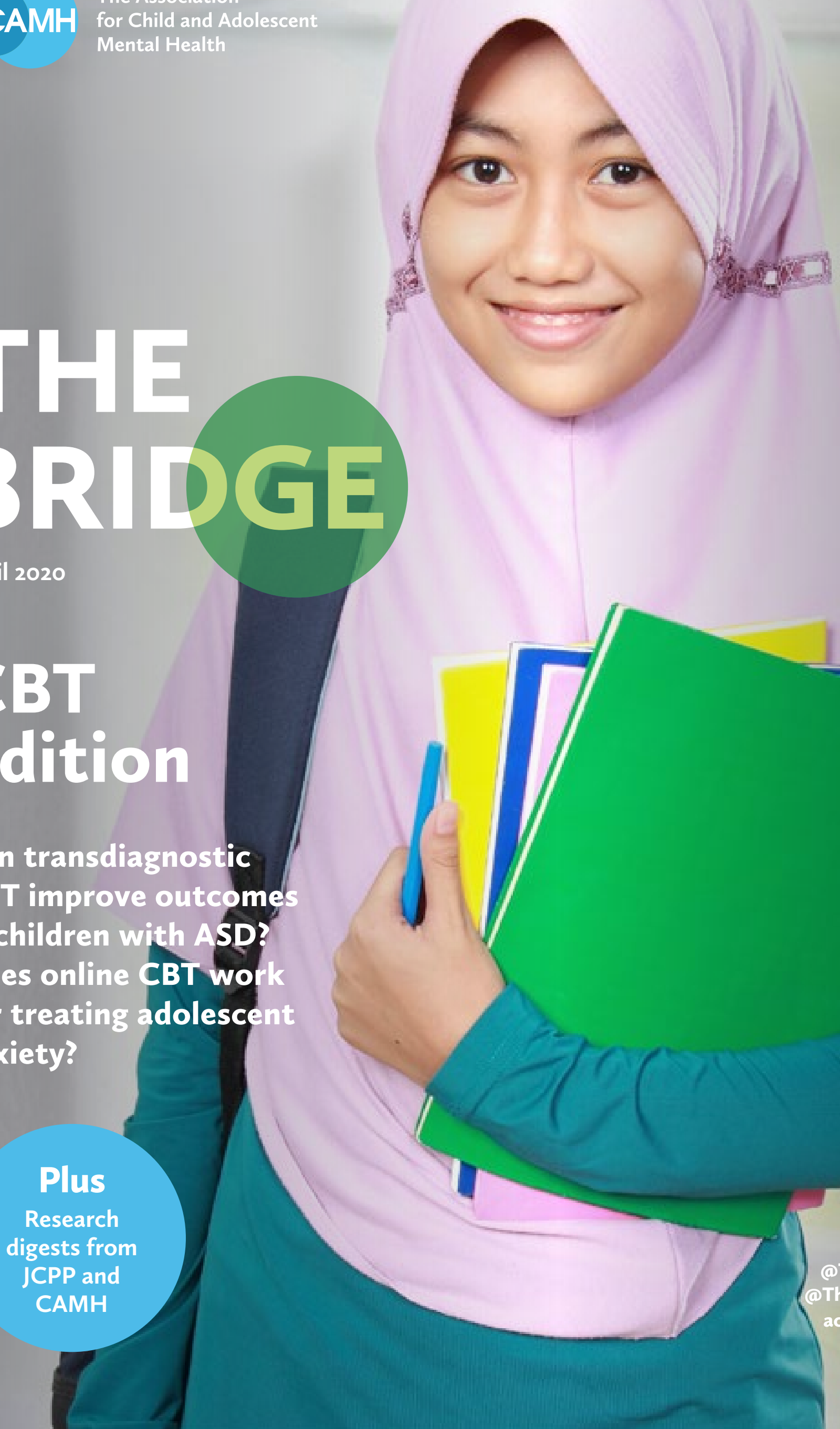


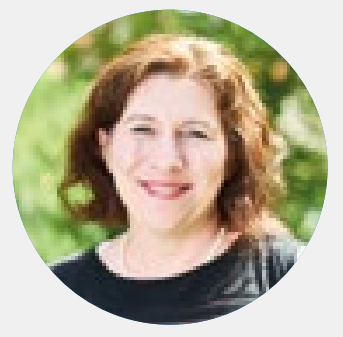

\section{Dr Juliette Kennedy}

\section{The Bridge Editor}

I am Dr. Juliette Kennedy, Editor of The Bridge, and a Consultant Child and Adolescent Psychiatrist working clinically in a North Yorkshire CAMHS team. I am Associate Director of Medical Education in the trust I work in, also Training Program director for CAMHS higher training in Yorkshire.

The Bridge presents the most clinically-relevant research from our two peer-reviewed journals: Child and Adolescent Mental Health and The Journal of Child Psychology and Psychiatry, as well as interesting and important studies from the wider literature. Please let us know what you'd like to see in upcoming editions by sending an email to me at: researchdigests@acamh.org

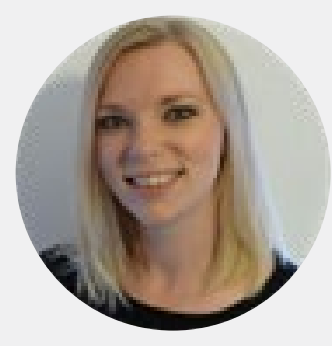

\section{Dr Jessica K. Edwards}

Research highlights in this edition are prepared by $\mathrm{Dr}$ Jessica K. Edwards. Jessica is a freelance editor and science writer, and started writing for 'The Bridge' in December 2017.

\section{Contents:}

P3 A day in the life of a CWP

P5 A thinner cortex predicts a better response to $\mathrm{CBT}$

p6 CYP-IAPT - Where next?

p9 Online CBT is ineffective for treating adolescent anxiety

p1o Can transdiagnostic CBT improve outcomes in children with ASD?

pil CBT and sertraline are effective treatments for paediatric anxiety, but how do they work? 


\section{A day in the life of a CWP}

\section{By Susan Moore}

A children's wellbeing practitioner (CWP) is a highly specialist role in a CAMHS team. CWPs deliver low-intensity psychological interventions for mild to moderate low mood and anxiety disorders. We treat children and young people using a variety of interventions such as:

- Behavioural Activation

- Graded Exposure

- Worry management

- Parent-led CBT

\section{Initial Assessments}

A psychological wellbeing practitioner (PWP) will start the therapeutic process by offering an initial assessment, in order to gather more information about the presenting problem. This is an important part of the process as it allows the PWP to develop a CWP formulation, which helps with moving onto the intervention stage (Curry, Dunsmuir \& Fuggle's, 2012).

A CWP's initial assessment is different to a generic CAMHS clinician. It has a tight structure and a short time slot. The initial assessment can be broken down into 3 sections: information gathering, information giving and shared decision making (Reach Out).

The information gathering section of the assessment can be broken down into four key elements: 4 W's, 5 areas formulation, impact and risk. The 4 W's explore the presenting problems in terms of; what is the problem? where does it happen? with who is the problem better or worse and when does this happen? These four brief questions allow for the practitioner to be time efficient in this area of the assessment (Richards \& Whytes, 2011). Following the 4 W's we complete a 5 areas formulation, identify impact and complete a risk assessment.

According to the "Reach Out" document, the next steps after completing the information gathering section are to complete a problem statement, create patient-centred goals and give treatment information (Richards \& Whytes, 2011). A problem statement draws a conclusion to the initial assessment. I try to encourage the young person to write their own problem statement with some verbal help from myself. Once a problem statement is completed we can then think about setting goals and the intervention we are going to use.

\section{Typical day as a CWP}

Following the initial assessment we will meet with the young person to complete a collaborative 5 areas formulation. During this session we also review the RCADS and start the joint decision-making approach to decide which intervention we are going to use.

A typical day for a CWP will always include a number of 30 minute intervention slots. We usually see our patients weekly or fortnightly. Preparation is needed for these appointments as they often rely on the use of worksheets for homework/tasks. We see the young person for around 30 minutes which allows for us to provide feedback to the parents/carers. Once we have done this feedback we have a brief window to add a case note and make any other changes.

\section{Summary of a typical intervention - BA for depression}

- Treatment Session I (with parents): Young person and parents (1 hour). The CWP provides psychoeducation about depression. We will also discuss the treatment rationale with a brief personalised exploration of the model (Homework-Activity monitoring form). We will complete RCADS.

- Treatment Session 2 (30 minutes): A review of the daily monitoring form with a treatment rationale review. During this session we will look at what activities provide positive and negative reinforcement and consider the balance of activities. (Homework is continued activity monitoring and balancing of activities).

- Treatment Session 3 (30 Minutes): A review of daily monitoring forms and activity targets. We will then complete a values-based assessment task where we look at different areas in the young person's life such as family, friends, hobbies, self-care, future plans and physical health. We will then generate one activity to try. (Homework is to review the diary exercise and introduce one activity target).

- Treatment Session 4 (30 minutes): A review of the values-based activity task. Then, the generation of a list of activities to inform activity scheduling. We will commence activity planning and scheduling using ACE logs and activity scheduling sheets. (Homework is 3 activity targets).

- Treatment Session 5: A review of the daily monitoring form. We continue activity planning and scheduling using ACE logs and activity scheduling sheets. (Homework is 3 activity targets). 
- Treatment Session 6 (with parents 1 hour): A review of progress and continued activity planning and scheduling, using ACE logs and activity scheduling sheets, with some problem solving. (Homework is to add or remove or adjust activities based on learning).

- Treatment Session 7: Continue activity planning and scheduling using ACE logs and activity scheduling sheets. Problem solving and thinking about any areas left to work on. (Homework is to add or remove or adjust activities based on learning).

- Session 8 and beyond (with parents). We complete a relapse prevention exercise. A review of learning and accomplishments. CWP will provide advice: including top tips for staying well.

- Discharge - planning for the end of treatment (with parents). Complete progress review and finalise relapse prevention plan. Complete RCADS again.

\section{A Typical CWP's Diary is below:}

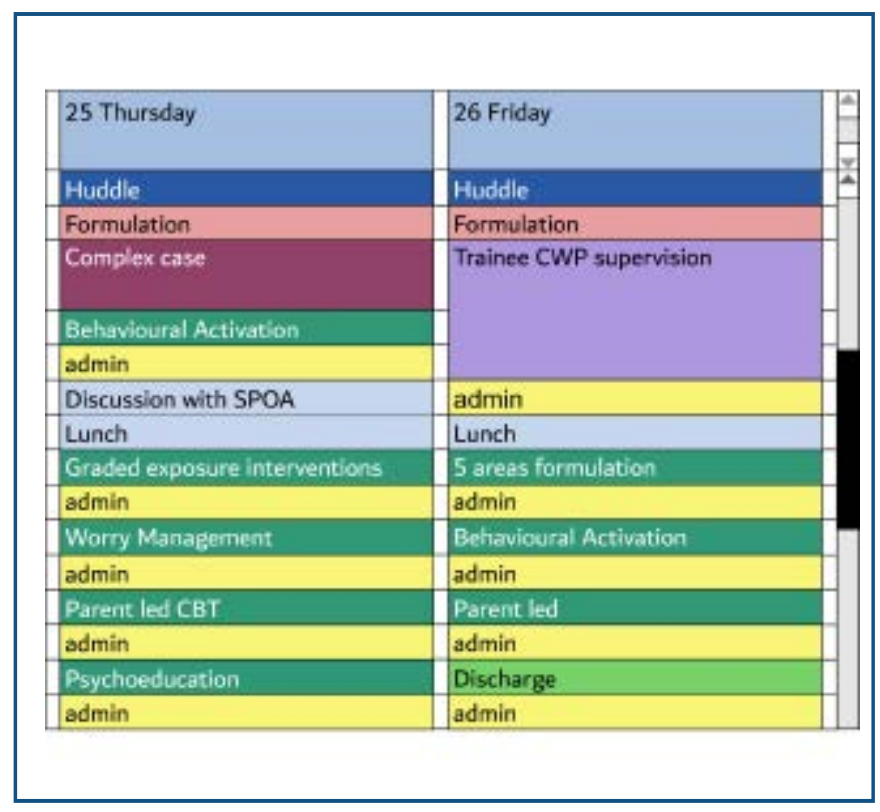

\section{Providing supervision}

Another part of our role as a qualified CWP is to provide clinical and caseload management to trainee CWPs. This supervision is weekly as trainees have a set number of hours of required supervision for their course. Supervision is to ensure that trainees have access to suitable cases and to check that they are able to stick to their evidenced-based model of treatment.

\section{Working alongside Single Point of Access to CAMHS}

As CWPs we work very closely with the Single Point of Access Team to ensure that our initial assessment are selected to be mild to moderate in risk. This ensures that chosen cases benefit from our specific structured model. A CWP model may not be appropriate if there is complexity or risk or if there are neurodevelopmental concerns that need to be assessed.

\section{Formulation and team working}

As a CWP we have weekly formulation slots in our diary. Clinicians can book a half hour slot to discuss a young person that they would like to refer to us for low intensity work. During this discussion a CWP will think about previous work undertaken, risk and complexity. A CWP will work with children and young people who need further work, after completing a tier 3 CAMHS intervention.

Once a young person has completed a low intensity intervention such as BA, the CWP can then think about other interventions that the young person may benefit from such as Graded Exposure.

If a CWP is worried about a child, and thinks they may need higher intensity work, we can discuss this with the tier 3 team through supervision, formulation or case discussion.

\section{Attending complex case discussion}

As part of working in a generic CAMHS team, a CWP will attend a weekly complex case discussion. The form of the complex case discussion depends on what the clinician who is presenting wants from the meeting. One aim may be to think about a plan/future work for the young person. A CWP can also take a case to discuss. They may discuss a case that has gone well or a case that they feel they need support with.

I think it's important for CWPs to take cases that have gone well, to help other clinicians in the team understand the structured role of the CWP. A CWP may also contribute with ideas about how a low intensity intervention may benefit the young person under discussion.

\section{Working with complex cases as a low intensity worker}

As discussed, a CWP will work with young people with mild to moderate (current or historical) risk to do a specific piece of work to help meet the goals of the young person. The lead professional continues to hold the case and manage risk whilst the CWP offers their intervention.

\section{Referring to:}

Curry, V. Dunsmuir, S. Fuggle, P. (2013). CBT with Children, Young People and Families. London: S. 63-64.

Richards, D. Whyte, M.. (2008). Reach Out. Available: https://cedar.exeter.ac.uk/media/ universityofexeter/schoolofpsychology/cedar/ documents/Reach_Out_3rd_edition.pdf. Last accessed 06/03/20. 


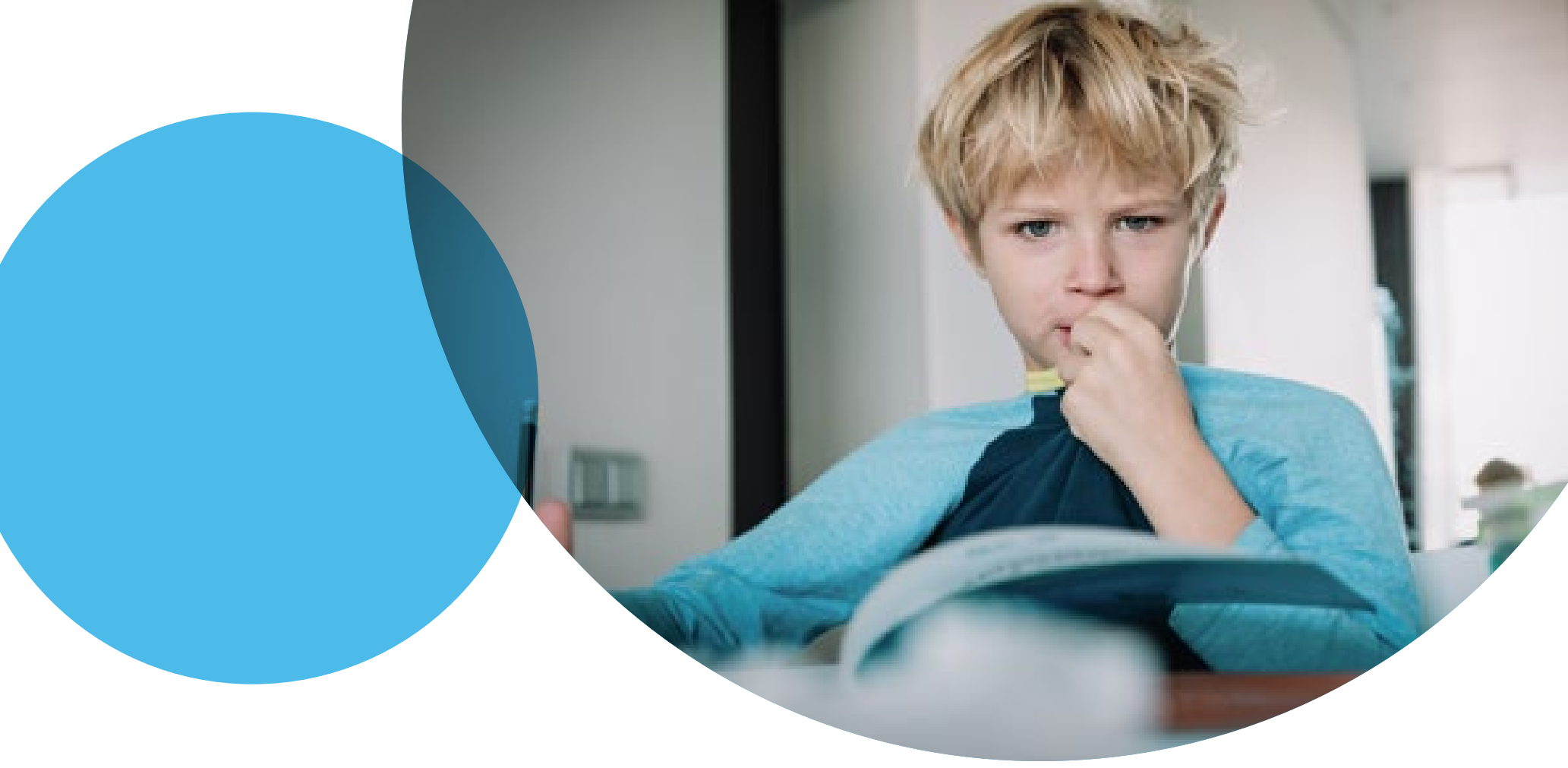

\title{
A thinner cortex predicts a better response to CBT
}

\author{
By Jessica K. Edwards
}

David Pagliaccio and colleagues in the USA have used magnetic resonance imaging (MRI) to identify neural markers that might predict a child's response to treatment for obsessive-compulsive disorder (OCD). The researchers recruited 28 unmedicated children with OCD and 27 matched healthy participants, and conducted MRI at baseline to estimate the sub-cortical volumes and cortical thicknesses in the brain. The patients with $O C D$ then completed 12-16 cognitivebehavioural therapy (CBT) sessions and the intervention outcomes were correlated with the imaging data.

The researchers found that children with $O C D$ exhibited reduced white matter connectivity in brain networks that typically support cognitive control processes (i.e., the ability to control one's thoughts and behaviours) compared with healthy children. They also identified that the thickness of several regions in these control networks predicted a patient's response to therapy. In short, the thinner the cortices, the better responses these children had to CBT.

"As treatment for OCD can be expensive and timeconsuming, and given that a portion of patients will not always respond, finding markers of who is likely to respond could help to guide treatment selection", explains Pagliaccio. "Because these identified regions that support control processes tend to thin over typical development, our findings might suggest that advanced cortical maturation may relate to a better CBT response. Our identification of pre-treatment neural markers could, therefore, help to identify patients most likely to respond to exposure-based therapy or other treatments in the future". The researchers are now conducting a larger study of younger children with $O C D$ to identify neural risk markers for OCD development.

\section{Referring to:}

Pagliaccio, D., Cha, J., He, X., Cyr, M., YanesLukin, P., Goldberg, P., Fontaine, M., Rynn, M.A. and Marsh, R. (2019), Structural neural markers of response to cognitive behavioural therapy in paediatric obsessive-compulsive disorder. J. Child Psychol. Psychiatr. doi: 10.111/jcpp.13191.

\section{Glossary:}

Cognitive-behavioural therapy: a form of talking therapy that encourages patients to manage their psycho-social problems by changing the way they think and behave; CBT focuses on current problems and finds practical ways to improve state-of-mind on a day-by-day basis. 


\title{
CYP-IAPT - Where next?
}

\author{
By Dr Markku Wood, Consultant Clinical Psychologist, BABCP Accredited, HEA Fellow
}

In this article I will outline for the reader the Children and Young People's Improving Access to Psychological Therapies project (CYP-IAPT), including where it came from, where we are now, and the challenges we have faced on the way.

In keeping with the associated literature for this edition of The Bridge, this article will focus on those areas in CYP-IAPT that have utilised CBT for children and young people (CYP) and their families. Finally, the article will discuss the future of this ambitious project and how we can continue to carry on delivering evidence-based therapies to as many CYP and families as need it.

\section{What is CYP-IAPT and where did it come from?}

CYP-IAPT is an innovative and transformational project undertaken by Health Education England (HEE), NHS England (NHSE), multiple Children's Mental Health Services (NHS and Voluntary Sector), Higher Education Institutions across England and, now in our newest developments, Department for Education and Local Authority/Educational institutions. The focus is on transforming mental health services for CYP and their families (DoH \& DfE, 2018).

Now in its seventh year the initial aims of the project were (and continue to be) to improve mental health services for CYP and their families through 5 core principles (CAMHS press, 2014):
1. To improve accessibility of mental health services to all those who need it.

2. To improve participation in the development and running of CYP-MH services by those who use the services.

3. To increase the use of Routine Outcome Measures (ROMS) so that CYP services can more reliably measure change/improvements in distressing symptomology.

4. To develop a (non-blaming) culture of accountability in CYP-MH services.

5. To develop the range of evidence-based psychological therapies that are delivered in CYP-MH services, and for them to be delivered by qualified and accredited psychological therapists.

Principle 5 will be the key focus of this article and is the one I am most passionate about as the Clinical Lead for Northumbria University's CYP-IAPT programmes of training for practitioners, and also as a Consultant Clinical Psychologist working in CAMHS in the NHS.

The transformative component of the CYP-IAPT initiative took the key evidence-based therapies (based on NICE guidelines) to develop Post Graduate (full and part-time) training programs including several therapeutic modalities from systemic, to interpersonal therapy, parenting interventions, to counselling. Although these are all key evidenced-based modalities this article will focus on CBT-based therapies including: 
- High intensity Cognitive Behavioural Therapy (HI-CBT).

- Low intensity CBT (LI-CBT) based interventions, delivered in $\mathrm{MH}$ services and schools within the Educational Mental Health Practitioner (EMHP) and Children's Wellbeing Practitioner (CWP) roles.

As a transformative project, programmes were also developed to train those who provide supervision and leadership in CYP-MH services, so that the project could have an impact at all levels in CYP-MH services.

\section{Where are we now?}

Having now trained thousands of therapists across the country, the aim of the CYP-IAPT initiative is now to move from one of transformation to one of 'business as usual'. In theory most CYP-MH services should now have plenty of highly trained staff to deliver evidencebased therapies across England. This considered, the focus has now moved to early intervention. The newly developed roles of CWP and EMHP work into both CYP-MH services and schools respectively to identify those CYP who are at risk of developing, or have already developed, mild to moderate symptoms of anxiety and depression. These innovative roles aim to prevent CYP needing to access tertiary $\mathrm{MH}$ services as they can deliver highly evidenced (CBT-based), manualised interventions to CYP and their families. These practitioners are highly trained and closely supervised/assessed to ensure fidelity to the specialised psychological interventions, which include:

- Behavioural activation (BA).

- Parent-led CBT (PL-CBT).

- Graded Exposure and several other anxiety-based interventions.

In addition to these interventions, CWP and EMHP practitioners are also trained to quickly assess the key needs for the CYP based on Routine Outcome Measures (ROMS), clinical expertise, and most critically on the goals the CYP and their family set.

As discussed above, there is not enough scope within this article to explore the highly evidenced-based therapeutic modalities that are non CBT-based, nor is there scope to discuss all of the CBT-based interventions that are delivered under the umbrella of CYP-IAPT. With that in mind, I will focus on two particularly exciting developments in CYP-MH, BA and PL-CBT.

\section{Behavioural Activation}

Behaviour activation is a discreet intervention which has origins in Behavioural theory and CBT (NHS, 2018). In $B A$ the patient is encouraged, not simply to increase activity levels, but after a detailed functional assessment they are supported to engage in activities, which are more likely to positively reinforce closeness to others, enjoyment, and a sense of achievement.

A recent thought-provoking randomised controlled non-inferior trial looked at a (heavily evidenced-based) more traditional CBT approach for depression versus behavioural activation in the treatment of major depressive disorder (Richards et al., 2016). Richards et al's (2016) study found similar efficacy in both conditions, however, the crucial difference is that BA can be delivered by more junior staff and on a less intensive basis, meaning that it can be delivered by well trained, but less experienced team members.

Studies such as this clearly indicate that LI CBT practitioners can be used in a cost effective way. Although the "COBRA" (Richards et al, 2016) study was conducted with adults, there is now a clear and expanding evidence base for the effective use of $B A$ with CYP (Pass et al. 2018; 2018).

\section{Parent-led CBT}

It is a common misconception that CBT can only be delivered on a one to one basis; however, there is an ever-increasing evidence base for the use of CBT methods with families (Friedberg, 2006). Parentled CBT is a manualised intervention which aims to train parents to support their children to overcome anxiety. Parents are taught simple questioning styles and behavioural techniques which allow their CYP to engage more actively in challenging negative thoughts and changing toxic behaviours (Brittnay et al., 2017) the approach developing a strong evidence base (Thirlwall, Cooper, Creswell, 2017) and is used in CYP-IAPT programmes. Like BA, PL-CBT is effective when delivered by less experienced staff, supporting further the role for LI-CBT practitioners.

\section{Challenges faced}

Despite training thousands of $\mathrm{HI}$ and $\mathrm{LI}$ practitioners across England, it is clear that $\mathrm{MH}$ services are still finding it a challenge to utilise these highly trained specialists. Anecdotal feedback gathered from CYP-IAPT trainees and course leads across the country suggests that, upon returning full time to their hosting service, practitioners are unable to complete specialist interventions but instead they are returning to generic CYP mental health roles. This could be due to a multitude of reasons and is the subject of several prospective studies for our team. 
Feedback from trainees and course leaders across the country suggests that resource pressures, lack of understanding of CYP-IAPT in leadership, and lack of appropriate supervision may be some of the key contributors to CYP-IAPT trainees not using their newly acquired skills.

Lack of supervision and little opportunity for practitioners to continue using their therapeutic skills poses further risks too. It is well documented that effective therapy requires competent supervision (Alfonsson, S., Parling, T., Spännargård, A., Andersson, G. \& Lundgren, T., 2018) and without this there is a risk of digression from fidelity to the therapeutic model.

Finally, problems with resourcing can pose a risk to the efficacy of therapy, forcing practitioners to see the CYP less often than recommended or for fewer sessions than recommended, leaving them open to delivering a 'sub-therapeutic dose' of therapy.

\section{Where are we going?}

Having now trained thousands of $\mathrm{HI}$ and $\mathrm{LI}$ therapists across England, it is time to make sure that all of these highly trained specialists are utilised effectively. By making clear adjustments to the infrastructure of CYP-MH services to accommodate these specialist practitioners, initial results from national evaluations show the efficacy and improvements in outcomes for our CYP and their families (Fuggle et al 2019). By embracing the LI practitioner roles, services stand to gain in the amount of CYP they can effectively treat and free up space for the $\mathrm{HI}$ practitioners to see those CYP who need more intensive work.

\section{Dr Markku Wood}

Markku is a highly experienced clinical

psychologist, with extensive experience of working across a variety of mental health difficulties, developmental disorders with individuals, couples, and families. He currently works as a Senior Lecturer in Psychological Therapies at Northumbria University and in private practice. Also having worked as Clinical Lead/Principal Clinical Psychologist for an NHS adult mental health service in Cambridgeshire and a Senior Clinical Psychologist for an NHS Child and adolescent mental health service and Early Intervention for Psychosis service in Norfolk.

\section{Referring to:}

Transforming children and young people's mental health provision: a green paper (2017 and 2018).

Department of Health and Social Care and Department for Education

CYP IAPT principles in Child \& Adolescent Mental Health services, values and standards

"Delivering With and Delivering Well" (2014). CAMHS Press ISBN 978-0-9572096-9-5.

Types of Talking Therapies (2018) https://www. nhs.uk/conditions/stress-anxiety-depression/ types-of-therapy.

D.A., Ekers, D. McMillan, D., Byford, S,. \& Warren FC, (2016). Cost and Outcome of Behavioural Activation versus Cognitive Behavioural Therapy for Depression (COBRA): a randomised, controlled, non-inferiority trial. DOI:https://doi. org/10.1016/So140-6736(16)31140-0.

Friedberg R.D. (2006) A Cognitive-Behavioral Approach to Family Therapy. Journal Contemporary Psychotherapy 36:159-165. DOI 10.1007/s10879-006-9020-2.

Brittany M. Rudy, B.M., Zavrou, S., Johnco, C., Storch, E.A. \& Lewin (2017). Parent-Led Exposure Therapy: A Pilot Study of a Brief Behavioral Treatment for Anxiety in Young Children: Journal of Child and Family Studies volume 26, pages2475-2484(2017).

Thirlwall K., Cooper P., Creswell C., (2017) Guided parent-delivered cognitive behavioral therapy for childhood anxiety: Predictors of treatment response, Journal of Anxiety Disorders. Volume 45, January 2017, Pages 43-48.

Alfonsson, S., Parling, T., Spännargård, A., Andersson, G. \& Lundgren, T. (2018) The effects of clinical supervision on supervisees and patients in cognitive behavioral therapy: a systematic review. Cognitive Behaviour Therapy. Volume 47, - Issue 3 206-228

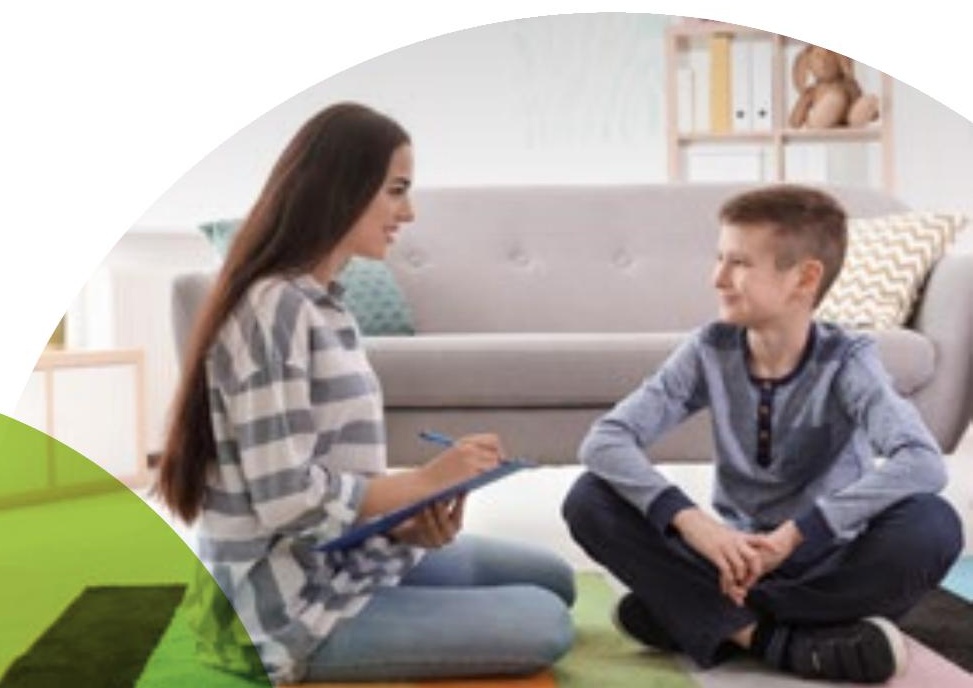




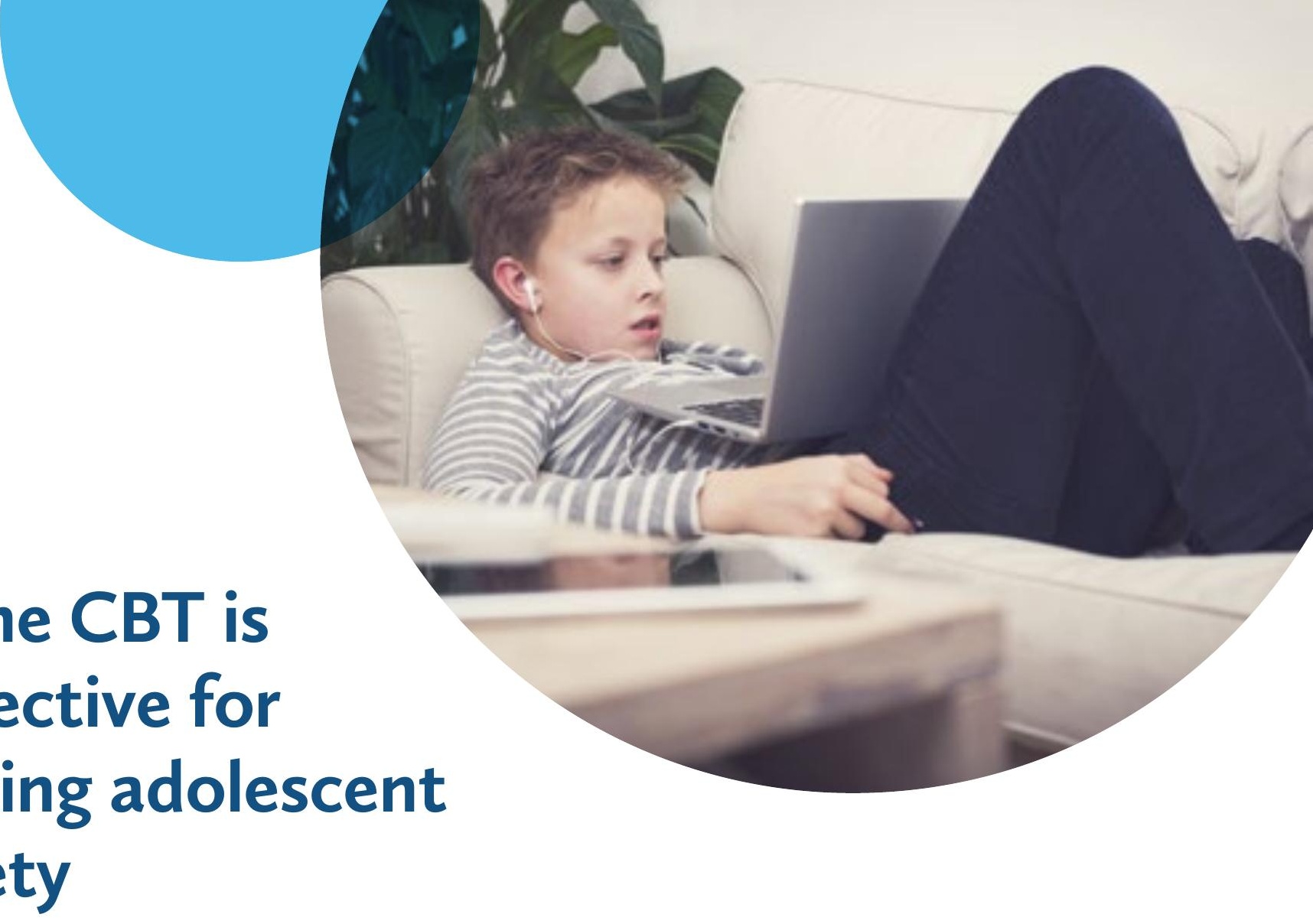

By Jessica K. Edwards

Researchers in Reading, UK have assessed whether self-completed, therapist-supported online cognitive behavioural therapy (CBT) for adolescent anxiety disorders is an effective treatment approach. Data obtained from 60 adolescents showed that there is no difference in patient outcomes between those receiving the "BRAVE for Teenagers-ONLINE" treatment ${ }^{1}$ and waitlist controls. There was also no improvement in outcomes when parents also received online sessions. While this data needs to be replicated in future studies, these findings suggest that this online CBT treatment, with or without parent sessions, is ineffective at treating anxiety disorders. Although the treatment uptake by adolescents with anxiety is low, and waiting times for treatment are often high, ${ }^{2,3}$ the researchers propose that routine use of this online therapy by clinical services to help overcome anxiety issues is premature.

\section{Referring to:}

Waite, P., Marshall, T. \& Creswell C. (2019), A randomized trial of internet-delivered cognitive behaviour therapy for adolescent anxiety disorders in a routine clinical care setting with and without parent sessions. Child Adolesc. Ment. Health. 24: 242-250. doi: 10.111/camh.12311.

\section{References:}

${ }^{1}$ Spence, S.H. et al. (2006), BRAVE for TeenagersONLINE: An Internet based program for adolescents with anxiety. Brisbane, Australia: University of Queensland.

${ }^{2}$ Merikangas, K.R. et al. (2011), Service utilization for lifetime mental disorders in U.S. adolescents: Results of the National Comorbidity SurveyAdolescent supplement (NSC-A). J. Am. Acad. Child Adolesc. Psychiatry 50: 32-45. doi: 10.1016/j. jaac.2010.10.006.

${ }^{3}$ Frith, E. (2016), CentreForum Commission on Children and young people's mental health: State of the nation. Centreforum. Available from: http://centreforum.org/live/wp-content/ uploads/ 2016/04/State-of-the-Nation-report-web.pdf.

\section{Glossary:}

Cognitive-behavioural therapy: a form of talking therapy that encourages patients to manage their psycho-social problems by changing the way they think and behave; CBT focuses on current problems and finds practical ways to improve state-of-mind on a day-by-day basis.

\section{See also:}

https://www.brave-online.com/ 


\title{
CBT and sertraline are effective treatments for paediatric anxiety, but how do they work?
}

\author{
By Jessica K. Edwards
}

Data from randomized controlled trials $(\mathrm{RCTs})^{1}$ support that cognitive-behavioural therapy (CBT), selective serotonin reuptake inhibitor (SSRI) treatment (Sertraline) and a combination of both are effective at treating anxiety disorders. How these evidence-based treatments exert their effects, however, is unknown. In 2019, Matti Cervin and colleagues analysed data from the largest RCT for paediatric anxiety disorders ${ }^{2}$ to examine how these treatments affect different domains of anxiety (i.e., anxiety severity, frequency, manifestations, and avoidance strategies).

In their unique approach, Cervin et al. conceptualized youth anxiety as a network of interconnected nodes, with each node corresponding to one aspect of anxiety. They then analyzed which aspects of this anxiety network were directly affected by evidencebased treatments for youth anxiety. Consistent with prior studies, ${ }^{2}$ the researchers found that combined CBT with the SSRI sertraline had strong effects on the anxiety network over placebo treatment. These treatments seemed to predominantly attenuate the severity of anxiety feelings and impairments in the home setting. No direct effects were found in relation to impairments outside of the home, such as at school.

"Current treatments might be improved by collaborating more with school personnel and by thoroughly assessing how a young individual with anxiety is affected in school", explains Cervin. "Although we analyzed data from the largest treatment study conducted to date, we still need to see whether our results replicate; if they do, then treatment strategies that readily address anxiety-associated school impairments should be developed and tested".

\section{Referring to:}

Cervin, M., Storch, E.A., Piacentini, J., Birmaher, B., Compton, S.N., Albano, A.M., Gosch, E., Walkup, J.T. \& Kendall, P.C. (2019), Symptom-specific effects of cognitive-behavioural therapy, sertraline, and their combination in a large randomized controlled trial of paediatric anxiety disorders. J. Child Psychol. Psychiatr. doi: 10.111/jcpp.13124.

\section{References:}

'Wang, Z. et al. (2017), Comparative effectiveness and safety of cognitive behavioural therapy and pharmacotherapy for childhood anxiety disorders: A systematic review and meta-analysis. JAMA Pediatr. 171: 1049-1056. doi: 10.1001/ jamapediatrics.2017.3036.

${ }^{2}$ Walkup, J.T. et al. (2008), Cognitive behavioural therapy, sertraline, or a combination in childhood anxiety. N. Eng. J. Med. 359: 2753-2766. doi: 10.1056/NEJMoao804633

\section{Glossary:}

Randomized controlled trial: an experimental setup whereby participants are randomly allocated to an intervention/treatment group or a control/ placebo group; randomization of participants occurs after assessments for eligibility, and is used to minimize selection bias.

Cognitive-behavioural therapy: a form of talking therapy that encourages patients to manage their psycho-social problems by changing the way they think and behave; CBT focuses on current problems and finds practical ways to improve state-of-mind on a day-by-day basis.

Selective serotonin reuptake inhibitors: a class of drug that is typically used as an antidepressant in the treatment of anxiety or major depressive disorders. These drugs increase circulating serotonin levels by limiting its reabsorption into the cell; the effect is an increased level of serotonin available to bind postsynaptic receptors and increased serotonin-mediated neurotransmission.

\section{See also:}

Child/Adolescent Anxiety Multimodal Study, NCToo052078: clinicaltrials.gov/ct2/show/ NCTooo 2078. 